PROCEEDINGS OF THE

AMERICAN MATHEMATICAL SOCIETY

Volume 128, Number 6, Pages 1641-1646

S 0002-9939(99)05419-2

Article electronically published on September 30, 1999

\title{
NONVANISHING OF SYMMETRIC SQUARE $L$-FUNCTIONS OF CUSP FORMS INSIDE THE CRITICAL STRIP
}

\author{
WINFRIED KOHNEN AND JYOTI SENGUPTA
}

(Communicated by Dennis A. Hejhal)

\begin{abstract}
We shall give a certain nonvanishing result for the symmetric square $L$-function of an elliptic cuspidal Hecke eigenform w.r.t. the full modular group inside the critical strip.
\end{abstract}

\section{INTRODUCTION}

Let $f$ be a normalized cuspidal Hecke eigenform of integral weight $k$ on the full modular group $S L_{2}(\mathbf{Z})$ and denote by $D_{f}^{*}(s)(s \in \mathbf{C})$ the symmetric square $L$-function of $f$ completed with its archimedean $\Gamma$-factors. As is well-known [7], [8], $D_{f}^{*}(s)$ has a holomorphic continuation to $\mathbf{C}$ and is invariant under $s \mapsto 2 k-1-s$. Note that, by [3], $D_{f}^{*}(s)$ (up to a variable shift) also is the standard zeta function of a cuspidal automorphic representation of $G L(3)$, and so by [4] zeros of $D_{f}^{*}(s)$ can occur only inside the critical strip $k-1<\operatorname{Re}(s)<k$. According to the generalized Riemann hypothesis, the zeros of $D_{f}^{*}(s)$ should all lie on the critical line $\operatorname{Re}(s)=k-\frac{1}{2}$.

The last statement of course is far from being settled. On the other hand, it turns out to be comparatively easy to prove nonvanishing results for $D_{f}^{*}(s)$ on the average. For example, in [6] Xian-Jin Li used an approximate functional equation for an average sum of the $D_{f}^{*}(s)$ to show that for any given $s$ with $k-1<\operatorname{Re}(s)<$ $k, s \neq k-\frac{1}{2}, \zeta(s-k+1) \neq 0$, there are infinitely many different $f$ such that $D_{f}^{*}(s)$ is not zero.

In the present note, using a different approach we will prove that given any $s$ with $k-1<\operatorname{Re}(s)<k, \operatorname{Re}(s) \neq k-\frac{1}{2}$, then for all $k$ large enough there exists a Hecke eigenform $f$ of weight $k$ such that $D_{f}^{*}(s) \neq 0$. For the proof we use a "kernel function" for $D_{f}^{*}(s)$ as given by Zagier in [8] and then proceed in a similar way as in [5], where a corresponding result for Hecke $L$-functions was proved.

\section{NOTATION}

For $s \in \mathbf{C}$ we usually write $s=\sigma+i t$ with $\sigma, t \in \mathbf{R}$.

Received by the editors July 31, 1998 .

2000 Mathematics Subject Classification. Primary 11Fxx.

(C)2000 American Mathematical Society 


\section{Statement of Result}

Let $k$ be an even integer $\geq 12$ and let $S_{k}$ be the space of cusp forms of weight $k$ w.r.t. the full modular group $\Gamma_{1}=S L_{2}(\mathbf{Z})$, equipped with the usual Petersson scalar product $\langle$,$\rangle . For f(z)=\sum_{n \geq 1} a(n) e^{2 \pi i n z}(z \in \mathcal{H}=$ upper half plane $)$ a normalized Hecke eigenform in $S_{k}$ (recall that normalized means $a(1)=1$ ), we denote by

$$
D_{f}(s)=\prod_{p}\left(1-\alpha_{p}^{2} p^{-s}\right)^{-1}\left(1-\alpha_{p} \beta_{p} p^{-s}\right)^{-1}\left(1-\beta_{p}^{2} p^{-s}\right)^{-1} \quad(\sigma>k)
$$

the symmetric square $L$-function of $f$, where the product is taken over all rational primes $p$ and $\alpha_{p}, \beta_{p}$ are defined by

$$
\alpha_{p}+\beta_{p}=a(p), \alpha_{p} \beta_{p}=p^{k-1} .
$$

By [7, [8, $D_{f}(s)$ has a holomorphic continuation to $\mathbf{C}$, and the function

$$
D_{f}^{*}(s)=2^{-s} \pi^{-3 s / 2} \Gamma(s) \Gamma\left(\frac{s-k+2}{2}\right) D_{f}(s)
$$

satisfies the functional equation

$$
D_{f}^{*}(2 k-1-s)=D_{f}^{*}(s) .
$$

Let $\left\{f_{k, 1}, \ldots, f_{k, g_{k}}\right\}\left(g_{k}=\operatorname{dim} S_{k}\right)$ be the basis of normalized Hecke eigenforms of $S_{k}$.

Theorem. Let $t_{0} \in \mathbf{R}$ and $0<\epsilon<\frac{1}{2}$. Then there exists a positive constant $C\left(t_{0}, \epsilon\right)$ depending only on $t_{0}$ and $\epsilon$ such that for $k>C\left(t_{0}, \epsilon\right)$ the function

$$
\sum_{\nu=1}^{g_{k}} \frac{1}{\left\langle f_{k, \nu}, f_{k, \nu}\right\rangle} D_{f_{k, \nu}}^{*}(s)
$$

does not vanish at any point $s=\sigma+i t_{0}, k-1<\sigma<k-\frac{1}{2}-\epsilon, k-\frac{1}{2}+\epsilon<\sigma<k$.

Corollary. Let $s \in \mathbf{C}$ be fixed with $k-1<\sigma<k, \sigma \neq k-\frac{1}{2}$. Then for all $k$ large enough there exists a normalized Hecke eigenform $f$ in $S_{k}$ such that $D_{f}^{*}(s) \neq 0$.

\section{ProOF}

The proof proceeds along similar lines as in [5]. We consider the cusp forms dual w.r.t. the Petersson scalar product to the values $D_{f}^{*}(s)(2-k<\sigma<k-1)$ where $f$ is any normalized Hecke eigenform in $S_{k}$. These have been constructed by Zagier [8] and will be denoted $\Phi_{s}$ as in [8] in what follows. To state the relevant properties of $\Phi_{s}$, we need to introduce several notations.

Let $\Delta$ be a discriminant, i.e. $\Delta \in \mathbf{Z}$ and $\Delta \equiv 0,1(\bmod 4)$. Put

$$
L(s, \Delta)= \begin{cases}\zeta(2 s-1), & \text { if } \Delta=0 \\ L_{D}(s) \sum_{d \mid f, d>0} \mu(d)\left(\frac{D}{d}\right) d^{-s} \sigma_{1-2 s}\left(\frac{f}{d}\right), & \text { if } \Delta \neq 0\end{cases}
$$

where if $\Delta \neq 0$ we have written $\Delta=D f^{2}$ with $f \in \mathbf{N}$ and $D$ the discriminant of $\mathbf{Q}(\sqrt{\Delta}),\left(\frac{D}{.}\right)$ is the Kronecker symbol, $L_{D}(s)$ the associated $L$-function defined by analytic continuation of the series $\sum_{n>1}\left(\frac{D}{n}\right) n^{-s}(\sigma>1), \mu$ is the Möbius function and $\sigma_{\nu}(m)=\sum_{d \mid m, d>0} d^{\nu}(m \in \mathbf{N}, \nu \in \mathbf{C})$. 
Furthermore, for $t$ an integer with $\Delta<t^{2}$ and $s \in \mathbf{C}$ with $\frac{1}{2}<\sigma<k$ we define

$$
\begin{gathered}
I_{k}(\Delta, t ; s)=\int_{0}^{\infty} \int_{-\infty}^{\infty} \frac{y^{k+s-2}}{\left(x^{2}+y^{2}+i t y-\frac{1}{4} \Delta\right)^{k}} d x d y \\
\quad=\frac{\Gamma\left(k-\frac{1}{2}\right) \Gamma\left(\frac{1}{2}\right)}{\Gamma(k)} \int_{0}^{\infty} \frac{y^{k+s-2}}{\left(y^{2}+i t y-\frac{1}{4} \Delta\right)^{k-\frac{1}{2}}} d y
\end{gathered}
$$

where the second integral converges absolutely for $1-k<\sigma<k$ if $\Delta \neq 0$ [8] Proposition 4]. We are now in a position to state Zagier's theorem.

Theorem ([8]). Let $k \geq 4$ be an even integer. For $m \in \mathbf{N}, s \in \mathbf{C}$ set

$$
\begin{aligned}
c_{m}(s) & =m^{k-1} \sum_{t \in \mathbf{Z}}\left(I_{k}\left(t^{2}-4 m, t ; s\right)+I_{k}\left(t^{2}-4 m,-t ; s\right)\right) L\left(s, t^{2}-4 m\right) \\
+ & \begin{cases}\frac{(-1)^{\frac{k}{2}} \Gamma(s+k-1) \zeta(2 s)}{2^{2 s+k-3} \pi^{s-1} \Gamma(k)} u^{k-s-1}, & \text { if } m=u^{2}, u>0, \\
0, & \text { if } m \text { is not a perfect square. }\end{cases}
\end{aligned}
$$

Then the following assertions hold:

i) The series converges absolutely and uniformly for $2-k<\sigma<k-1$.

ii) The function

$$
\Phi_{s}(z)=\sum_{m \geq 1} c_{m}(s) e^{2 \pi i m z} \quad(z \in \mathcal{H}, 2-k<\sigma<k-1)
$$

is in $S_{k}$.

iii) Let $f$ be a normalized Hecke eigenform in $S_{k}$. Then the Petersson scalar product of $\Phi_{s}$ and $f$ is given by

$$
\left\langle\Phi_{s}, f\right\rangle=c_{k} \frac{\pi^{\frac{1}{2}(s+k-1)}}{2^{s+k-1} \Gamma\left(\frac{1+s}{2}\right)} D_{f}^{*}(s+k-1)
$$

where

$$
c_{k}=\frac{(-1)^{\frac{k}{2}} \pi}{2^{k-3}(k-1)} .
$$

From the theorem, taking $m=1$ we deduce

$$
\begin{gathered}
c_{1}(s)=c_{k} \frac{\pi^{\frac{1}{2}(s+k-1)}}{2^{s+k-1} \Gamma\left(\frac{1+s}{2}\right)} \sum_{\nu=1}^{g_{k}} \frac{1}{\left\langle f_{k, \nu}, f_{k, \nu}\right\rangle} D_{f_{k, \nu}}^{*}(s+k-1) \\
(2-k<\sigma<k-1) .
\end{gathered}
$$

In view of the functional equation (1), it is sufficient to prove the theorem in the range $k-\frac{1}{2}+\epsilon<\sigma<k$. Suppose that the right-hand side of (3) vanishes at $s=\frac{1}{2}+\delta+i t_{0}$ where $\epsilon<\delta<\frac{1}{2}$. Then from the definition of $c_{1}(s)$ we obtain

$$
\begin{aligned}
\sum_{t \in \mathbf{Z}}\left(I _ { k } \left(t^{2}-4, t\right.\right. & \left.\left.; \frac{1}{2}+\delta+i t_{0}\right)+I_{k}\left(t^{2}-4,-t ; \frac{1}{2}+\delta+i t_{0}\right)\right) L\left(\frac{1}{2}+\delta+i t_{0}, t^{2}-4\right) \\
& +(-1)^{\frac{k}{2}} \frac{\Gamma\left(k-\frac{1}{2}+\delta+i t_{0}\right) \zeta\left(1+2 \delta+2 i t_{0}\right)}{2^{k-2+2 \delta+2 i t_{0}} \pi^{-\frac{1}{2}+\delta+i t_{0}} \Gamma(k)}=0
\end{aligned}
$$


or

$$
\begin{gathered}
\frac{(-1)^{\frac{k}{2}-1} 2^{k} \Gamma(k)}{\Gamma\left(k-\frac{1}{2}+\delta+i t_{0}\right)} \sum_{t \in \mathbf{Z}}\left(I_{k}\left(t^{2}-4, t ; \frac{1}{2}+\delta+i t_{0}\right)+I_{k}\left(t^{2}-4,-t ; \frac{1}{2}+\delta+i t_{0}\right)\right) \\
\cdot L\left(\frac{1}{2}+\delta+i t_{0}, t^{2}-4\right)=\frac{\zeta\left(1+2 \delta+2 i t_{0}\right)}{2^{2 \delta-2+2 i t_{0}} \pi^{-\frac{1}{2}+\delta+i t_{0}}}
\end{gathered}
$$

Clearly the right-hand side of (4) does not depend on $k$ and is never zero for $\epsilon \leq \delta \leq \frac{1}{2}$. Therefore in absolute value it is bounded from below by a positive absolute constant depending only on $\epsilon$.

We will show that the left-hand side of (4) goes to zero uniformly for $\epsilon<\delta<\frac{1}{2}$ as $k \rightarrow \infty$, thereby arriving at a contradiction.

We first look at the terms $I_{k}\left(t^{2}-4, t ; \frac{1}{2}+\delta+i t_{0}\right)+I_{k}\left(t^{2}-4,-t ; \frac{1}{2}+\delta+i t_{0}\right)$ in (4).

If $t=0$, we obtain from $(2)$

$$
\begin{gathered}
2 I_{k}\left(-4,0 ; \frac{1}{2}+\delta+i t_{0}\right)=2 \frac{\Gamma\left(k-\frac{1}{2}\right) \Gamma\left(\frac{1}{2}\right)}{\Gamma(k)} \int_{0}^{\infty} \frac{y^{k-\frac{3}{2}+\delta+i t_{0}}}{\left(y^{2}+1\right)^{k-\frac{1}{2}}} d y \\
=\frac{\Gamma\left(k-\frac{1}{2}\right) \Gamma\left(\frac{1}{2}\right)}{\Gamma(k)} B\left(\frac{k-\frac{1}{2}+\delta+i t_{0}}{2}, \frac{k-\frac{1}{2}-\delta-i t_{0}}{2}\right)
\end{gathered}
$$

where $B(z, w)$ is the Beta function and in the last line we have used [1, 6.2.1]. Since $B(z, w)=\frac{\Gamma(z) \Gamma(w)}{\Gamma(z+w)}$ we deduce

$$
2 I_{k}\left(-4,0 ; \frac{1}{2}+\delta+i t_{0}\right)=\sqrt{\pi} \frac{\Gamma\left(\frac{k-\frac{1}{2}+\delta+i t_{0}}{2}\right) \Gamma\left(\frac{k-\frac{1}{2}-\delta-i t_{0}}{2}\right)}{\Gamma(k)} .
$$

Next suppose that $t \neq 0, \pm 2$. We will then use the fact that $I_{k}\left(t^{2}-4, t ; s\right)$ can be expressed in terms of standard Legendre functions $P_{\nu}^{\mu}(z)$ [즈, Proof of Proposition 4]. More precisely, one has

$I_{k}\left(t^{2}-4, t ; s\right)=\left(\frac{\left|t^{2}-4\right|}{4}\right)^{\frac{s-k}{2}} \frac{\Gamma\left(k-\frac{1}{2}\right) \sqrt{\pi}}{\Gamma(k)} \cdot \begin{cases}I_{k, s}\left(\frac{i t}{\sqrt{\left|t^{2}-4\right|}}\right), & \text { if } t^{2}<4 \\ e^{\frac{\pi i}{2}(s-k) \operatorname{sign}(t)} I_{k, s}\left(\frac{|t|}{\sqrt{t^{2}-4}}\right), & \text { if } t^{2}>4\end{cases}$

where

$$
\begin{gathered}
I_{k, s}(z)=\frac{2^{1-k} \sqrt{\pi}}{\Gamma\left(k-\frac{1}{2}\right)} \Gamma(k-1+s) \Gamma(k-s)\left(z^{2}-1\right)^{-\frac{k-1}{2}} P_{-s}^{1-k}(z) \\
(1-k<\sigma<k, z \in \mathbf{C} \backslash(-\infty, 1]) \text {. Also for }|z-1|<2 \text { the identity } \\
P_{-s}^{1-k}(z)=\frac{1}{\Gamma(k)}\left(\frac{z+1}{z-1}\right)^{\frac{1-k}{2}} F\left(s, 1-s, k ; \frac{1-z}{2}\right)
\end{gathered}
$$

holds, where $F(a, b, c ; z)(|z|<1)$ denotes the Gauss hypergeometric series [1, 8.1.2].

Therefore for $t= \pm 1$ we easily find that

$$
\begin{gathered}
2\left(I_{k}\left(-3,1 ; \frac{1}{2}+\delta+i t_{0}\right)+I_{k}\left(-3,-1 ; \frac{1}{2}+\delta+i t_{0}\right)\right) \\
\ll_{t_{0}} \frac{\left|\Gamma\left(k-\frac{1}{2}+\delta+i t_{0}\right) \Gamma\left(k-\frac{1}{2}-\delta-i t_{0}\right)\right|}{2^{k} \Gamma(k)^{2}},
\end{gathered}
$$


and for $\pm t \geq 3$ we get

$$
\begin{gathered}
2\left(I_{k}\left(t^{2}-4, t ; \frac{1}{2}+\delta+i t_{0}\right)+I_{k}\left(t^{2}-4,-t ; \frac{1}{2}+\delta+i t_{0}\right)\right) \ll_{t_{0}}\left(t^{2}-4\right)^{-\frac{1}{4}+\frac{\delta}{2}} \\
\cdot\left(\frac{|t|-\sqrt{t^{2}-4}}{|t|+\sqrt{t^{2}-4}}\right)^{\frac{k-1}{2}} \frac{\left|\Gamma\left(k-\frac{1}{2}+\delta+i t_{0}\right) \Gamma\left(k-\frac{1}{2}-\delta-i t_{0}\right)\right|}{2^{k} \Gamma(k)^{2}}
\end{gathered}
$$

where the constants implied in $\ll_{t_{0}}$ depend only on $t_{0}$.

Finally, for $t= \pm 2$ we have by [8, Proposition 4]

$$
\begin{gathered}
2\left(I_{k}\left(0,2 ; \frac{1}{2}+\delta+i t_{0}\right)+I_{k}\left(0,-2 ; \frac{1}{2}+\delta+i t_{0}\right)\right) \\
=2\left(e^{\frac{\pi i}{2}\left(\frac{1}{2}-k+\delta+i t_{0}\right)}+e^{-\frac{\pi i}{2}\left(\frac{1}{2}-k+\delta+i t_{0}\right)}\right) \sqrt{\pi} \\
\cdot \frac{\Gamma\left(\delta+i t_{0}\right) \Gamma\left(k-\frac{1}{2}-\delta-i t_{0}\right)}{\Gamma(k)} 2^{-k+\frac{1}{2}+\delta+i t_{0}} \\
\ll_{t_{0}, \epsilon} \frac{\left|\Gamma\left(k-\frac{1}{2}-\delta-i t_{0}\right)\right|}{2^{k} \Gamma(k)} .
\end{gathered}
$$

We now look at the quantities $L\left(\frac{1}{2}+\delta+i t_{0}, t^{2}-4\right)$ on the left-hand side of (4). For $|t|=2$, we have by definition

$$
L\left(\frac{1}{2}+\delta+i t_{0}, 0\right)=\zeta\left(2 \delta+2 i t_{0}\right)
$$

which is a continuous function in the range $\epsilon \leq \delta \leq \frac{1}{2}$, provided $t_{0} \neq 0$. If $t_{0}=0$, the same applies to the function

$$
2 i^{k} \cos \left(\frac{\pi}{2}\left(\frac{1}{2}+\delta+i t_{0}\right)\right) \cdot \zeta\left(2 \delta+2 i t_{0}\right)
$$

where

$$
2 i^{k} \cos \left(\frac{\pi}{2}\left(\frac{1}{2}+\delta+i t_{0}\right)\right)=e^{\frac{\pi i}{2}\left(\frac{1}{2}-k+\delta+i t_{0}\right)}+e^{-\frac{\pi i}{2}\left(\frac{1}{2}-k+\delta+i t_{0}\right)}
$$

is the second factor on the right-hand side of $(8)$.

On the other hand, if $|t| \neq 2$, then for all $\delta \geq 0$ and all $\epsilon^{\prime}>0$ one has

$$
L\left(\frac{1}{2}+\delta+i t_{0}, t^{2}-4\right) \ll_{t_{0}, \epsilon^{\prime}}\left|t^{2}-4\right|^{\frac{1}{2}+\epsilon^{\prime}} .
$$

In fact, if $t^{2}-4$ is a fundamental discriminant, this follows from [2, Chapter 12, Example $22(\mathrm{~b})$ ], and the general case then easily follows from the definitions.

Denote the left-hand side of (4) by $L_{k, \delta, t_{0}}$. Then from (5)-(9) (fixing any small $\epsilon^{\prime}>0$ in (9)) and the separate discussion in the case $|t|=2, t_{0}=0$ above we deduce that

$$
\left|L_{k, \delta, t_{0}}\right| \ll_{t_{0}, \epsilon}\left|\frac{2^{k} \Gamma\left(\frac{k-\frac{1}{2}+\delta+i t_{0}}{2}\right) \Gamma\left(\frac{k-\frac{1}{2}-\delta-i t_{0}}{2}\right)}{\Gamma\left(k-\frac{1}{2}+\delta+i t_{0}\right)}\right|+\frac{\left|\Gamma\left(k-\frac{1}{2}-\delta-i t_{0}\right)\right|}{\Gamma(k)}
$$

$$
+\left|\frac{\Gamma\left(k-\frac{1}{2}-\delta-i t_{0}\right)}{\Gamma\left(k-\frac{1}{2}+\delta+i t_{0}\right)}\right|+\frac{\left|\Gamma\left(k-\frac{1}{2}-\delta-i t_{0}\right)\right|}{\Gamma(k)} \sum_{t \geq 3}\left(t^{2}-4\right)^{\frac{1}{4}+\frac{\delta}{2}+\epsilon^{\prime}}\left(\frac{t-\sqrt{t^{2}-4}}{t+\sqrt{t^{2}-4}}\right)^{\frac{k-1}{2}} .
$$

Elementary considerations show that the sum over $t \geq 3$ converges and is bounded by an absolute constant independent of $k$. 
Note that by Legendre's duplication formula for the $\Gamma$-function, the first term on the right-hand side of (10) can also be written in the form

Using the fact that

$$
2^{\frac{3}{2}-\delta} \sqrt{\pi}\left|\frac{\Gamma\left(\frac{k}{2}-\frac{1}{4}-\frac{\delta}{2}-\frac{i t_{0}}{2}\right)}{\Gamma\left(\frac{k}{2}+\frac{1}{4}+\frac{\delta}{2}+\frac{i t_{0}}{2}\right)}\right| .
$$

$$
\lim _{x \rightarrow \infty} x^{b-a} \frac{\Gamma(x+a)}{\Gamma(x+b)}=1 \quad(a, b \in \mathbf{C} \backslash \mathbf{R} ; x \rightarrow \infty)
$$

[1, 6.1.46, 6.1.47], we now see indeed that

$$
L_{k, \delta, t_{0}} \rightarrow 0 \quad(k \rightarrow \infty) .
$$

Moreover, using more precisely the explicit asymptotics of $x^{b-a} \frac{\Gamma(x+a)}{\Gamma(x+b)}$ for $x \rightarrow \infty$ given in 1, 6.1.47], one sees that the convergence in (11) is uniform in $\delta$, since $\delta>\epsilon>0$ is bounded away from zero.

This proves the theorem.

\section{REFERENCES}

[1] N. Abramowitz and I. Stegun: "Handbook of Mathematical Functions", Dover, New York, 1965

[2] T. Apostol: "Introduction to Analytic Number Theory", Springer, Berin-Heidelberg-New York, 1976 MR 55:7892

[3] S. Gelbart and H. Jacquet: A relation between automorphic representations of $G L_{2}$ and $G L_{3}$, Ann. Sci. E.N.S. IV ser., 11, 471-542 (1978) MR 81e:10025

[4] H. Jacquet and J. A. Shalika: A nonvanishing theorem for zeta functions of $G L_{n}$, Invent. Math. 38, 1-16 (1976/77) MR 55:5583

[5] W. Kohnen: Nonvanishing of Hecke $L$-functions associated to cusp forms inside the critical strip, J. of Number Theory vol. 67, no. 2, 182-189 (1997) MR 98j:11037

[6] X.-J. Li: On the poles of Rankin-Selberg convolutions of modular forms, Trans. Amer. Math. Soc. 348, no. 3, 1213-1234 (1996) MR 96h:11038

[7] G. Shimura: On the holomorphy of certain Dirichlet series, Proc. London Math. Soc. 31, 75-98 (1975) MR 52:3064

[8] D. Zagier: Modular forms whose Fourier coefficients involve zeta-functions of quadratic fields, in "Modular Functions of One Variable VI" (eds. J.-P. Serre and D. Zagier), pp. 105-169, LNM no. 627, Springer, Berlin-Heidelberg-New York, 1976. MR 58:5525

Universität Heidelberg, Mathematisches Institut, Im Neuenheimer, Feld 288, D-69120 Heidelberg, Germany

E-mail address: winfried@mathi.uni-heidelberg.de

School of Mathematics, Tata Institute for Fundamental Research, Homi Bhabha ROAD, BOMBAY 400 005, INDIA

E-mail address: sengupta@math.tifr.res.in 